\title{
Sufficiency of Vertex Matrix Check for Robust Stability of Interval Matrices via the Concept of Qualitative Robustness
}

\author{
Rama K. Yedavalli and Nagini Devarakonda \\ Department of Mechanical and Aerospace \\ Engineering, The Ohio State University, \\ Columbus, Ohio 43210-1276 \\ yedavalli.1@osu.edu//ndevarak@gmail.com
}

\begin{abstract}
This paper revisits the issue of robust stability analysis of linear interval parameter matrices, which used to be a highly active research topic in the eighties and nineties. The reason for this revived interest in this topic is that the recent research by the authors on Qualitative Stability, a topic of interest in the field of population/community dynamics in ecology is shown to shed considerable insight with possible new results in the robust stability of matrix families. Thus in this paper, we expand on the two notions of robustness introduced recently by the authors, namely 'Qualitative Robustness' and 'Quantitative Robustness' and investigate their interdependence. Specifically, it is shown that for a class of matrix families with specified 'Qualitative Robustness' indices, it is sufficient to check the stability of only 'vertex' matrices (i.e. an extreme point solution) to guarantee the robust stability of the entire interval matrix family. This is indeed deemed important and significant because with this result, we can easily identify for which 'interval matrix families' we need to resort to more sophisticated stability check algorithms, and for which families we can get away with a ' vertex matrix check' (i.e. an 'extreme point solution'). It turns out that this class of 'qualitative stable' matrices that admit 'vertex solution' for its 'quantitative robustness' is quite large. Thus the results of this paper offer new insight into the nature of interactions and interconnections in a matrix family on its robust stability. Encouraged by the results of this paper, continued research is underway in using this interdependence of 'qualitative robustness' and 'quantitative robustness' in the design of robust controllers for engineering systems.
\end{abstract}

\section{InTROdUCTION AND PERSPECTIVE:}

In the engineering/mathematical sciences, the aspect of 'Robust Stability' of matrix families has been an active topic of research for many decades, especially in the eighties and early nineties[1],[2],[3]. This issue essentially is manifested in 'uncertain linear dynamic systems' with real parameter variations and arises in many applications of systems and control theory. When the system is described by linear state space representation, the plant matrix elements typically depend on some uncertain parameters which vary within a given bounded interval. We label these matrix families as 'interval parameter matrix families'. It is to be noted that in our description of matrix families, there is a subtle difference between 'Interval Parameter Matrix family' and 'Interval Matrix family'. In this notation, 'Interval Matrix family' is a special case of 'Interval Parameter Matrix family'. Put it another way, while the parameter space is always a 'hyperrectangle', the matrix space is a 'Polytope' in the case of 'Interval Parameter Matrix' family, whereas it is a 'hyperrectangle' as well in the case of an 'Interval Matrix' family. Thus the general 'polytope' of matrices becomes a 'hyperrectangle' in the Interval matrix case. These notions can be made more precise mathematically as follows. Consider the linear state space description

$$
\dot{x}(t)=A(q) x(t) \quad q \in Q
$$

where $x(t) \in R^{n}$ is the state variable vector and $q \in R^{r}$ is a vector of uncertain parameters $q_{i}$ varying in the prescribed compact set $Q$. Specifically, let the parameters $q_{i}$ be given apriori bounds as

$$
q_{i L} \leq q_{i} \leq q_{i U} \quad i=1,2, \ldots r
$$

Assuming linear dependent variations $q_{i}$ in the entries of $A(q)$, we can write the matrix $A(q)$ of ( 1$)$ as

$$
A(q)=A_{0}+\sum_{i=1}^{r} q_{i} A_{i}
$$

where $A_{0}$ is the 'nominal' matrix and $A_{i}$ are constant, specified matrices, reflecting the 'structure' of the uncertainty. In this situation the set of possible $A(q)$ matrices $[A(q): q \varepsilon Q]$ forms a 'polytope' of matrices in $R^{n \times n}$. In other words, linear dependent variations $q_{i}$ in the entries of $A(q)$ produces a 'polytope' of matrices.

Now, we can specialize this situation for 'linear indepenedent variation' case. In this case, which we label as 'Interval Matrix' family, each individual element varies independently within a given interval. Thus the interval matrix family is denoted by

$$
A \in\left[A^{L}, A^{U}\right]
$$

as the set of all matrices $A$ that satisfy

$$
\left(A^{L}\right)_{i j} \leq A_{i j} \leq\left(A^{U}\right)_{i j} \text { for every } i, j
$$

In other words, the interval matrix family is a special case of the 'polytope' of matrices in the sense that the matrices $A_{i}$ above have only one nonzero positive element in a different location for each index $i$ and the nominal matrix $A_{0}$ is the matrix with all 'lower bounds' of the matrix elements i.e $A_{0}=A^{L}$. Thus an Interval matrix family can always be expressed as

$$
A=A^{L}+a_{1} E_{1}+a_{2} E_{2}+\ldots .+a_{r} E_{r} .
$$




$$
0 \leq a_{i} \leq a_{i u}, 1 \leq i \leq r
$$

where each matrix $E_{i}$ has only the single entry 1 at different location for different index $i$ and thus is of rank 1. We also notice that each $a_{i u}$ is a positive scalar. Let us now rearrange these positive scalars $a_{i u}$ in decreasing order of magnitude and label the matrices $E_{i}$ and the scalars $a_{i}$ such that they are labeled in this decreasing order. Thus, it is understood that

$$
a_{1 u} \geq a_{2 u} \geq a_{3 u} \geq \ldots \geq a_{r u}
$$

and similarly $E_{i}$ denotes that matrix with its single entry 1 being at that location corresponding to $a_{i}$ variation. Note that in the special case of an 'interval matrix' (i.e. linear independent variations), generated from the bounded interval set of (2), the number of vertex matrices, $h=2^{r}$ is an even number (exponent of two) where $r$ is the number of entries in the matrix that are considered uncertain. We see that the stability of these 'vertex' matrices $A^{i}$ is a necessary condition for the robust stability of this 'interval matrix' family. Assuming these 'vertex' matrices are Hurwitz stable, the issue of research is to analyze if every matrix within this family is also Hurwitz stable or not. Unfortunately, it is well known that the stability of vertex matrices is not a sufficient condition for the robust stability of the entire interval matrix family [1]. Till now in the literature, the special class of matrices which admit 'vertex matrix check' as a 'sufficient condition' for the robsut stability of the entire matrix family have been known to be the Metzlerian and M-matrices (which happen to be a class of nonnegative matrices) and symmetric matrix families and normal matrix families, and matrix families with all real eigenvalues[2]. However, some classes of these matrix families belong to the 'polytope' of matrices described above and these happen to be extremely restricted classes which are difficult to be realized in many practical applications. The only interval matrix family that admits 'vertex matrix check' is the $2 \times 2$ matrix family[4]. The objective of this paper is to expand the class of interval matrix families that admit 'vertex matrix' check as a sufficient condition for robust stability. The categorization of this class of matrix families is done with the help of conecpts from ecology. Towards this direction, we briefly review the two concepts introduced by the authors recently, namely 'qualitative robustness' and 'quantitative robustness' and use these ideas to show that for a certain class of matrix families with specified 'qualitative robustness indices', quantitaive robustness is achieved through the 'vertex matrix' check. With this backdrop the paper is organized as follows. In the next section, we briefly review some fundamental principles of ecology and establsih the rationale for this novel approach. Based on these principles, in section 3, the concept of 'Qualitative Robustness', as opposed to the traditional 'Quantitative Robustness', is introduced and it is shown that robustness can also be interpreted qualitatively in terms of the nature of 'interactions' and 'interconnections' of the system. Then the main result of how the eigenvalues of quantitative matrices with a specified set of 'qualitative robustness indices' are distributed is presented in section 4. Based on this result, finally in section 5, the class of matrix families which admit 'vertex matrix check' as a sufficient condition for robust stability are identified along with illustrative examples. Finally section 6 offers concluding remarks along with guidelines for future research in this exciting area.

\section{BRIEF REVIEW OF PRINCIPLES OF ECOLOGY}

The mathematical models of ecosystems are constructed based on the nature of the effect a species has on itself and its surrounding species [5], [6]. The sustenance or extinction of a species, apart from its own effect, is affected by its interactions with various other species in the ecosystem it belongs to. Hence the type of interaction is very critical to the sustenance of species. These interactions are classified as shown in Figure 1. Numerous models of ecological systems

$\left.\begin{array}{|l|l|l|}\hline \multicolumn{1}{|c|}{\begin{array}{c}\text { Interaction } \\ \text { type }\end{array}} & \begin{array}{c}\text { Digraph } \\ \text { representation }\end{array} & \begin{array}{c}\text { Matrix } \\ \text { representation }\end{array} \\ \hline \text { Cutualism } & {\left[\begin{array}{ll}* \\ +\end{array}\right.}\end{array}\right]$

Fig. 1. Types of interactions between species

have been proposed, an example of which is the set of nonlinear differential equations known as the Lotka-Volterra predator-prey models. Due to ambiguity in quantitative information of ecosystems, when these models are linearized, the Jacobian matrix resulting from linearization has entries with no numerical values but only the signs of the partial derivatives are known with certainty. In other words, in the Jacobian matrix, the 'qualitative' information about the species is represented by the signs,+- or 0 . Thus, the $(i, j)^{\text {th }}$ entry of the state space (Jacobian) matrix is simply ,+- , or 0 , with the ' + ' sign indicating species $j$ having a positive influence on species $i$, '-' sign indicating negative influence and ' 0 ' indicating no influence. The sign of a diagonal element gives information regarding the effect of a species on itself. In order to provide a better understanding of ecosystem dynamics, certain definitions pertaining to graph theory are presented below.

The qualitative Jacobian matrix can also be represented as a 'digraph' in terms of 'nodes' and 'paths'. For example, consider the $3 \times 3$ matrix $A$ in Figure 2. The numbered circles are the nodes (species) while the lines connecting the 
nodes are the paths (the off-diagonal elements representing the effect of one species on another). The sign $(+,-$ and 0)of a path indicates the nature of the effect and arrow represents the direction. Furthermore, products of paths(in

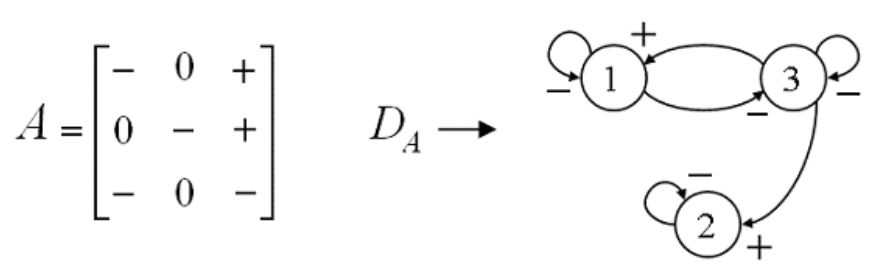

Fig. 2. A qualitative matrix and its corresponding digraph

matrix notation) with ordered indices are classified as follows [7]:

i. Product of off-diagonal elements of the form $a_{i j} a_{j i}$ connecting only two distinct nodes (indices) are known as ' $l$-cycles'. Since the product forms a cycle of indices, products $a_{12} a_{21}$ and $a_{21} a_{12}$ are considered to be the same.

ii. Product of off-diagonal elements of the form $a_{i j} a_{j k} \ldots a_{m i}$ connecting three or more nodes (indices) are known as ' $k$-cycles'. As is the case with $l$-cycles, the $k$-cycles $a_{12} a_{23} a_{31}$ and $a_{23} a_{31} a_{12}$ are considered to be the same.

In this research, we refer to $l$-cycles as interactions and to $k$-cycles as interconnections between species as depicted in Figure 3. The distinction between interactions and interconnections is that the former pertains to the relation between two species while the latter pertains to that between a group ( 3 or more) of species. Interactions and interconnections have significant effect on quantitative properties of matrices such as eigenvalue distribution and normality/condition number [8], [9], [10].

\section{Ecological sign stability}

An extremely interesting property resulting from the influence of interactions and interconnections on system stability is that of Qualitative (Sign) Stability. Qualitative stability, which is independent of magnitudes, implies Hurwitz stability in the ordinary sense of engineering sciences. In other words, once a particular sign matrix is shown to be qualitative (sign) stable, the non-zero elements

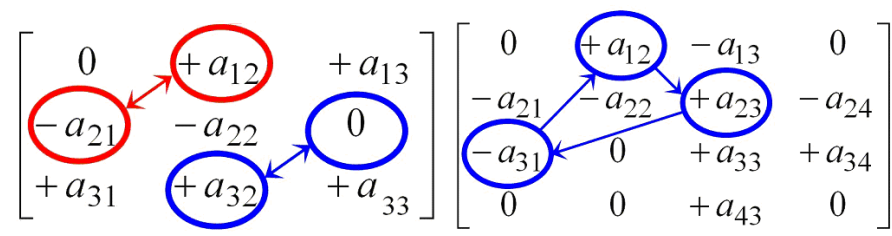

(a) Interactions

(b) Interconnections

Fig. 3. Interactions and interconnections in an ecosystem of this matrix can assume any value and for all those values the matrix is automatically Hurwitz stable. Therefore, a matrix $A$ is called 'sign stable' if each matrix $B$ of the same sign pattern as $A\left(\operatorname{sgn}\left(b_{i j}\right)=\operatorname{sgn}\left(a_{i j}\right) \forall i, j\right)$ is Hurwitz stable regardless of the magnitudes of $b_{i j}$.

This is the most attractive feature of a sign stable matrix: stability independent of magnitude. Thus, for a given dimension of the matrix, there are specific sign patterns (with + and - signs and zeros in specific locations of the matrix) which are 'Sign Stable'. In [9], an algorithm is presented which stores all the Sign Stable matrices of order 3, 4 and so on. The following $A, B$ matrices are examples of 'Sign Stable' matrices.

$$
A=\left[\begin{array}{ccc}
- & - & - \\
0 & - & 0 \\
+ & + & -
\end{array}\right] \quad B=\left[\begin{array}{cccc}
- & - & - & - \\
0 & - & - & 0 \\
0 & + & - & 0 \\
+ & 0 & + & -
\end{array}\right]
$$

It turns out from [11], [12], and [13], that all sign stable matrices contain only either 'predation-prey' or 'commensalism/ammensalism' interactions along with zeros at some very critical locations and combinations thereof in the entries of the dynamics matrix. It is thus shown that 'mutualism' and 'competition' interactions and nonzero interconnections are detrimental to qualitative stability. Sign stability has implications in robustness theory because this magnitude independence of stability imparts tolerance to perturbations thereby embracing the property of robustness. Equating a 'Sign Stable' system to a highly 'Qualitatively Robust' system, it can be concluded that system matrices with mutualism and competition interactions and nonzero interconnections are 'less qualitatively robust'. Based on this philosophy, we offer a 'qualitative robustness index' as a metric (or measure) of assessing robustness of linear uncertain dynamical systems.

\section{Qualitative RoBUstness}

As mentioned before it is clear that natural systems such as ecological and biological systems are highly robust under various perturbations. On the other hand, engineered systems can be made highly optimal for good performance but they tend to be non-robust under perturbations. Therefore, it is natural and essential for engineers to delve into the underlying features of natural systems which make them so robust and then apply these principles to make the engineered systems more robust. Toward this direction, the problem of assessment of qualitative robustness for linear uncertain systems is addressed from a completely new and refreshing framework of ecosystem dynamics. In these models, the interrelationship between various interactions and interconnections between states (species) play an extremely important role in making the overall system highly robust. It is proved and realized that certain interactions/interconnections enhance robustness while certain other interactions/interconnections destabilize the system [11], [12], [13]. Exploiting the property of 
qualitative stability derived from the study of interactions and interconnections in ecosystems, in what follows, we present new qualitative robustness assessment metrics namely,

$$
\begin{aligned}
& \beta_{1}=\frac{\text { no. of } a_{i i}>0}{\text { total } \text { no.of } a_{i i}(=n)} \\
& \beta_{2}=\frac{\text { no.of } l-\text { cycles }>0}{\text { total no.ofl }- \text { cycles }}
\end{aligned}
$$

We now introduce the following set of stable matrices which have $\beta_{1}=0$ and $\beta_{2}=0$, wherein it is assumed that the magnitudes of the elements are selected such that these matrices are all quantitatively (Hurwitz) stable.

Self Regulatory Full Predator-Prey Stable Matrices (SRF$P P S)$ In these stable matrices, we have $a_{i i}<0$ and $a_{i j} a_{j i}<0$. Self Regulatory Predator-Prey Ammensal Commensal Stable Matrices (SRPPACS) In these stable matrices, we have $a_{i i}<0$ and $a_{i j} a_{j i} \leq 0$.

SemiSelf Regulatory Full Predator-Prey Stable Matrices (SSRFPPS) In these stable matrices, we have $a_{i i} \leq 0$ and $a_{i j} a_{j i}<0$.

SemiSelf Regulatory Predator-Prey Ammensal Commensal Stable Matrices (SSRPPACS) In these stable matrices, we have $a_{i i} \leq 0$ and $a_{i j} a_{j i} \leq 0$.

Note that in the last two classes of Semi Self Regulatory matrices, we assume that there is at least one $a_{i i}$ which is negative and also that there are more Predator-Prey links than the Ammensal/Commensal combined. These assumptions assure that we avoid neutrally stable (all eigenvalues on the imaginary axis) situation. It is reiterated that all these four sets of matrices described above have both 'qualitative robustness indices', $\beta_{1}$ and $\beta_{2}$ to be equal to zero. In fact, for matrices of dimension 3 and above, we can introduce another qualitative robustness index $\beta_{3}$ as follows:

$$
\beta_{3}=\frac{\text { no.of } k-\text { cycles } \neq 0}{\text { total no.of } k-\text { cycles }}
$$

The reason we introduce index $\beta_{3}$ is that the Sign Stable (SS) matrices we described before have the property that for them, all the above three qualitative robustness indices are zero. Note that SS matrices could belong to either SRPPACS or SSRPPACS classes, but not to the SRFPPS, SSRFPPS classes because these latter matrices do not have any zero entries whereas the former class of matrices admit zero entries. Consider the four matrices $K, H, N$ and $S$ given below. Here matrix $K$ is an example of SRFPPS matrix, matrix $H$ is an example of SRPPACS, matrix $N$ is an example for SSRFPPS and finally matrix $S$ is an example of SSRPPACS matrix which also happens to be a Sign Stable (SS) matrix.

$$
K=\left[\begin{array}{cccc}
-3 & -1 & -0.8 & -4.8 \\
0.7 & -1.2 & -1.5 & -1.6 \\
1.3 & 2 & -0.4 & -0.5 \\
2.6 & 0.4 & 4 & -5.0 \\
-3 & -1 & -0.8 & -4.8 \\
0 & -1.2 & -1.5 & 0 \\
0 & 2 & -0.4 & 0 \\
2.6 & 0 & 4 & -5.0
\end{array}\right]
$$

$$
\begin{gathered}
N=\left[\begin{array}{cccc}
-3 & -1 & -0.8 & -4.8 \\
0.6 & -1.2 & -1.5 & -0.9 \\
0.7 & 2 & 0 & -0.40 \\
2.6 & 1.1 & 4 & -5.0
\end{array}\right] \\
S=\left[\begin{array}{cccc}
-3 & -1 & -0.8 & -4.8 \\
0 & -1.2 & -1.5 & 0 \\
0 & 2 & 0 & 0 \\
2.6 & 0 & 4 & -5.0
\end{array}\right]
\end{gathered}
$$

Using the above, we can also consider a cumulative measure $\beta$ given by $\beta=\beta_{1}+\beta_{2}+\beta_{3}$.

Note that under this definition, the 'higher' this index, the 'less robust' the system is qualitatively. It is also to be noted that it is relatively very easy to determine these robustness indices as the information needed for computing these indices is directly obtained from the dynamics matrix $A$. From the definition of $\beta_{i} \mathrm{~s}$, the minimum value of the cumulative index $\beta$ is 0 while the maximum value $\beta_{\max }=3$. Thus the farther this cumulative index is from zero, the less robust the system is. This implies that a matrix is qualitatively most robust when $\beta=0\left(\beta_{1}=\beta_{2}=\beta_{3}=0\right)$. Similarly, a matrix is least robust and is in fact unstable, when $\beta=\beta_{\max }=3$. Based on this definition a sufficient condition for stability is proposed as follows:

A quantitative matrix $A$ is always Hurwitz stable(unstable) if $\beta=0\left(\beta=\beta_{\max }=3\right)$. . It was shown in [14] that if a quantitative matrix has the sign pattern of a Sign Stable matrix, then for all the sign preserving variations in the matrix elements, one can have extremely large variations in those entries, making it 'enormously' robust for those sign preserving variations in those elements.

It can be easily seen that $\beta_{1}=1$ is a sufficient condition for instability. Thus, it is clear that the individual contribution of each $\beta_{i}$ also plays a significant role in qualitative robustness.

In the above four classes of matrices, their stability depends on the magnitudes of the elements of those matrices. Henceforth, we assume that the magnitudes are selected such that those matrices are Hurwitz stable.

Going forward, we club all these four classes of matrices, namely SRFPPS, SRPPACS, SSRFPPS and SSRPPACS matrices together as 'Ecological Stable Matrices'. It is important to emphasize that Ecological Stable matrices are those matrices for which the qualitative robustness indices $\beta_{1}$ and $\beta_{2}$ are zero. Thus Ecological Stable matrix class is much larger than Sign Stable matrix class.

\section{Eigenvalue Distribution of Ecological STABLE MATRICES}

In [9], it is proved that the real parts of the eigenvalues of Sign Stable matrices lie between the minimum and maximum diagonal elements of that matrix. In this paper, we expand on that property and introduce the concept of "stability degree inclusion by diagonal element". Recall that the magnitude of the real part of the dominant eigenvalue (the real part of the eigenvalue closest to the origin) is termed the stability degree and is denoted by $\alpha_{s}$. 
Stability degree inclusion by diagonal element: By stability degree inclusion by diagonal element, we mean the following:

$\alpha_{s}>\left|\left(a_{i i}\right)\right|_{\min }$

In other words, in the complex plane, the real part of the dominant eigenvalue lies to the left of the location of the least negative diagonal element of the matrix. Note that the above property is not enjoyed by many matrices especially with symmetric matrices. It is interesting to note that symmetric matrices always possess mutualism and/or competition links. Because of this, symmetric matrices never have the above property. However, it can be easily shown that the above described class of ecological stable matrices admit that property. Of course, whether a matrix with a fixed structure (i.e. with fixed placement/location of the PP, A and C links is Hurwitz stable or not and if it is Hurwitz stable, whether the above inclusion property is realized or not very much depends on the relative magnitudes of the predator-prey, ammensal, commensal links and their placement/ location in the matrix. In this paper, we focus on one special class of Ecological Stable matrices, namely SRFPPS matrices. From now on, we assume that for these SRFPPS matrices of a fixed link structure, the magnitudes of those links are determined such that they render that matrix Hurwitz stable with the inclusion property.

Conditions under which SRFPPS matrices Possess the property of 'Stability degree Inclusion by Diagonal Element': Note that for these SRFPPS matrices all $a_{i i}$ are negative and also each PP link product is also negative. Also the determinant expansion of a given $n$ - dimensional matrix contains the product terms of its elements as follows depending on whether $n$ is odd or even. For example when $\mathrm{n}$ is odd, it includes i) products of PP links (l-cycles) and diagonal elements, ii) product of all diagonal elements, iii) products of $\mathrm{k}$-cycles with diagonal elements where $k<n$ and finally iv) products of all k-cycles where $k=n$. When $\mathrm{n}$ is even, it also has additional terms of products of various PP links themselves. Because of their special nature, in the determinant expansion of SRFPPS matrices, it is easy to determine the sign of each of these terms and their summations. Let us denote the magnitude of summation of the terms involving only PP links (1-cycles) and diagonal elements as 'Strength of Interactions'. Similarly, let us denote the magnitudes of the summation of all terms involving $\mathrm{k}$ cycles as 'Strength of the Interconnections'. It is interesting to note that, for SRFPPS matrices, the 'Strength of Interactions' is linear with respect to the diagonal element, whereas the 'Strength of Interconnections' is not linear with respect to the diagonal element. For brevity, omitting the detailed mathematical proof, we present here the 'conceptual' proof. The property of 'Stability Degree inclusion by Diagonal Element' happens whenever the 'Strength of Interactions' is more than the 'Strength of the Interconnections'. In other words, whenever the' linear' term dominates the 'nonlinear' term (magnitude wise). It is interesting that this 'inclusion' property is lost when the 'Strength of Interconnections'(the nonlinear term) is more than the 'Strength of Interactions' (the linear term).

\section{SufFiciency of 'Vertex Matrix Stability CheCK' FOR Robust STABILITy OF ECOlOGICAL StABle InTERVAL MATRIX FAMILIES}

Motivated by the above discussion, we now focus our attention on interval matrix families that belong to the class of Ecological Stable matrices with a given fixed structure. Note that these matrix families are characterized by the qualitative robustness indices $\beta_{1}=0, \beta_{2}=0$ as well as a structure and magnitudes that render that matrix Hurwitz stable. Under the said constraints, it is quite easy to observe that for this class of Interval Matrices in which the parameter variation ranges are such that the corresponding vertex matrices each possess the property of Stability Degree Inclusion by Diagonal Element, the Hurwitz stability (and the associated inclusion property) of the vertex matrices is indeed a sufficient condition for the robust stability of that interval matrix family. This is due to the fact that for a given structure of the interaction links, the linear term of 'Strength of Interactions' is more dominant than the nonlinear term of 'Strength of Interconnections'.

Another interesting result is that if in addition to general Ecological Stable matrices if we focus on Sign Stable matrices (which happen to be a special case of Ecological Stable matrices with all three qualitative robustness indices $\beta_{1}=0, \beta_{2}=0, \beta_{3}=0$ ) then there is no need to even check the vertex matrix stability because as discussed in [14] Sign Stability becomes a sufficient condition for the entire interval matrix stability. Let us now illustrate the above main result of this paper with illustrative examples.

Example 1: Consider again, the 'interval matrix' family given by

$$
A=\left[\begin{array}{ccc}
a_{11} & a_{12} & 10 \\
-3 & -6 & 2 \\
-5 & -1 & -7
\end{array}\right]
$$

with the elements $a_{11}$ and $a_{12}$ being uncertain varying in some given intervals as follows:

$$
\begin{gathered}
-5 \leq a_{11} \leq-3 \\
10 \leq a_{12} \leq 12
\end{gathered}
$$

It can be easily seen that for the above matrix under the given parameter ranges, $\beta_{1}=0$ and $\beta_{2}=0$ throughout the interval matrix family. The four vertex matrices $A^{1}, A^{2}, A^{3}, A^{4}$ are given by

$$
\begin{aligned}
& A^{1}=\left[\begin{array}{ccc}
-5 & 10 & 10 \\
-3 & -6 & 2 \\
-5 & -1 & -7
\end{array}\right] \\
& A^{2}=\left[\begin{array}{ccc}
-3 & 10 & 10 \\
-3 & -6 & 2 \\
-5 & -1 & -7
\end{array}\right]
\end{aligned}
$$




$$
\begin{aligned}
A^{3} & =\left[\begin{array}{ccc}
-5 & 12 & 10 \\
-3 & -6 & 2 \\
-5 & -1 & -7
\end{array}\right] \\
A^{4} & =\left[\begin{array}{ccc}
-3 & 12 & 10 \\
-3 & -6 & 2 \\
-5 & -1 & -7
\end{array}\right]
\end{aligned}
$$

and

eig $\left(A^{1}\right)=-5.4054+9.0587 i,-5.4054-9.0587 i,-7.1891$, $e i g\left(A^{2}\right)=-4.4308+8.9504 i,-4.4308-8.9504 i,-7.1384$, eig $\left(A^{3}\right)=-5.3029+9.4052 i,-5.3029-9.4052 i,-7.3941$, eig $\left(A^{4}\right)=-4.3295+9.3107 i,-4.3295-9.3107 i,-7.3411$ From the above eigenvalue information it is clear that the vertex matrices are all not only Hurwitz stable but also satisfy the property of Stability Degree Inclusion by Diagonal Element. Therefore we can conclude that the above interval matrix family is Hurwitz stable.

In fact, it can be proven that in the case of SRFPPS matrix family, if the single vertex matrix containing the least magntitude diagonal element (subject to variation) and the highest strength PP links (subject to variations) has the 'inclusion' property then the entire interval matrix can be shown to be stable. In other words, a single special vertex matrix check is sufficient to guarantee the stability of the entire interval matrix family. In the above example, this special vertex matrix is $A^{4}$. So noting that $A^{4}$ possesses the 'inclusion' property, we can simply conclude that the entire interval matrix family is stable.

Example 2: Consider the interval matrix with two entries $\left(a_{11}\right.$ and $\left.a_{44}\right)$ varying in an interval as follows: $-1.5026 \leq$ $a_{11} \leq-0.5026$ and $-4.0026 \leq a_{44} \leq-1.5026$. This gives rise to the two bounding vertex matrices given by

$$
\begin{aligned}
A^{L} & =\left[\begin{array}{cccc}
-1.5026 & -12.06 & -0.06 & 0 \\
-0.25 & -0.0329 & 1 & 0.5 \\
0.25 & -4 & -1.0329 & 0 \\
0 & 0.5 & 0 & -4.0026
\end{array}\right] \\
A^{U} & =\left[\begin{array}{cccc}
-0.5026 & -12.06 & -0.06 & 0 \\
-0.25 & -0.0329 & 1 & 0.5 \\
0.25 & -4 & -1.0329 & 0 \\
0 & 0.5 & 0 & -1.5026
\end{array}\right]
\end{aligned}
$$

It can be seen that since $a_{24}=a_{42}=0.5$ which happens to be a mutalism link. Hence this family does not belong to Ecological Stable matrix family. Hence we cannot conclude that robust stability of vertex matrices is a sufficient condition for this family. Indeed, as discussed in [15] for this family robust stability of vertex matrices is not a sufficient condition as there are some unstable matrices in the interior of the family.

All the above examples clearly illustrate the importance of Ecological Stable matrices and the role of qualitative stability indices in the analysis of robust stability of interval matrices.

\section{Conclusions}

This paper brings about the union of two distinct concepts, namely the concept of Qualitative Stability (and the associated Qualitative Robustness aspect, a topic of interest in the field of Ecology), and Robust stability of Interval Matrices (and the associated Quantitative Robustness aspect, a topic of interest in Engineering Sciences) and develops a synergy between these two concepts by making qualitative robustness concept as a useful tool in analyzing the robust stability of a class of interval matrices. Specifically, it provides a condition under which for a special class of (labeled Ecological Stable) Matrix families, stability of Vertex matrices is a sufficient condition for the robust stability of the special interval matrix family. Note that this qualitative stability concept from ecology can be further exploited to solve many other interesting problems in engineering/mathematical sciences such as robust control design, critical parameter selection etc. Research is ongoing to address these interesting issues, thereby reviving interest in robust stability theory. It is anticipated that a result which says that for the special case of Ecological Stable matrices, convexity in stability exists (i.e. stability of vertex matrices is a sufficient condition for the stability of the entire interval matrix family) can be proven and is forthcoming.

\section{REFERENCES}

[1] B.R.Barmish, 1994. "New tools for robustness of linear systems". Macmillan Publishing Company.

[2] S.P.Bhattacharyya, Chapellat, H., and L.H.Keel, 1995. "Robust control: The parametric approach". Prentice Hall.

[3] Dorato, P., and Yedavalli, R. K., 1990. "Recent advances in robust control". IEEE Press.

[4] M.Mansour, 1989. "Robust stability of interval matrices". Proceedings of the IEEE CDC.

[5] Edelstein-Keshet, L., 1988. "Mathematical models in biology". McGraw Hill.

[6] Hofbauer, J., and Sigmund, K., 1988. "Growth rates and ecological models: Abc on ode". The Theory of Evolutions and Dynamical Systems, Cambridge University Press, London, pp. 29-59.

[7] Hogben, L., 2007. "Handbook of linear algebra". Discrete Mathematics and its Applications, Chapman and Hall/CRC.

[8] Yedavalli, R. K., and Devarakonda, N., 2009. "Qualitative principles of ecology and their implications in quantitative engineering systems". Proceedings of the ASME Annual Dynamic systems and control conference, DSCC, Oct, pp. 2009-2321.

[9] Yedavalli, R. K., and Devarakonda, N., 2010. "Sign stability concept of ecology for control design with aerospace applications". AIAA Journal of Guidance Control and Dynamics, 33(2), pp. 333-346.

[10] Devarakonda, N., and Yedavalli, R. K., 2010. "Engineering perspective of ecological sign stability and its application in control design". Proceedings of American Control Conference, Baltimore(MD), JuneJuly.

[11] Jeffries, C., 1974. "Qualitative stability and digraphs in model ecosystems”. Ecology, 55, pp. 1415-1419.

[12] Quirk, J., and Ruppert, R., 1965. "Qualitative economics and the stability of equilibrium". Reviews in Economic Studies, 32, pp. 311326.

[13] May, R., 1973. "Stability and complexity in model ecosystems". Princeton University Press, pp. Princeton, N.J.

[14] R.K.Yedavalli, 2006. "Qualitiative stability concept from ecology and its use in the robust stability analysis of linear interval parameter systems". Proceedings of the 5th IFAC Symposium on Robust Control Design (ROCOND06), Toulouse, France, July.

[15] Yedavalli, R. K., 2009. "Robust stability of linear interval parameter matrix family problem revisited with accurate representation and solution". Proceedings of the American Control Conference, St. Louis(MO), June, pp. 3710-3717. 